\title{
Large Eddy Simulation of an industrial gas-turbine combustion chamber using the sub-grid PDF method
}

\author{
G. Bulat ${ }^{\mathrm{a}}$, W. P. Jones ${ }^{\mathrm{b}, *}$, A. J. Marquis ${ }^{\mathrm{b}}$ \\ ${ }^{a}$ Siemens Industrial Turbomachinery Ltd, Waterside South, Lincoln LN5 7FD, UK \\ ${ }^{b}$ Department of Mechanical Engineering, Imperial College London, \\ Exhibition Road, London SW' $2 A Z, U K$
}

\begin{abstract}
An industrial gas turbine combustion chamber operating at a pressure of 3 bar is simulated using the $s g s-p d f$ evolution equation approach in conjunction with the Eulerian stochastic field solution method in the context of Large Eddy Simulation. A dynamic version of the Smagorinsky model is adopted for the sub-grid stresses and eight stochastic fields were utilised to characterize the influence of the sub-grid fluctuations. The chemistry was represented by an ARM reduced GRI 3.0 mechanism with 15 reaction steps and 19 species. The results show good agreement with the experimental data in the flame region at different axial locations. The measured NO emission levels are also reproduced to a good accuracy by the method. The results serve to demonstrate that simulations of complex combustion problems using detailed, but reduced, chemistry in industrial geometries are achievable.
\end{abstract}

Keywords: Gas Turbines, Large Eddy Simulation, premixed combustion, swirling flows.

\footnotetext{
Word count - text 4116 words

Word count - references 396 word

Word count - figures 1694 words

Word count - total (Method 2): 6206 words
} 


\section{Introduction}

The turbulent premixed flames to be found in industrial gas turbine combustors are difficult to study due to high levels turbulence, fast chemistry and complex geometrical features. Most of these devices operate at high pressure, which adds to the difficulty of obtaining experimental data and accounting for pressure effects in computational models. Large Eddy Simulation (LES) is a powerful and promising modelling technique particularly for highly swirling and unsteady flows. In the case of LES of turbulent combustion, account must be taken of the interactions between turbulence and the chemical reactions taking place. The main difficulties encountered in achieving this arise from the filtered chemical source terms, which represents the net rate of species formation and consumption through chemical reaction. Since these reactions are highly non-linear, the filtered values of the fields of chemical species mass fraction and temperature are strongly influenced by the sub-grid scale (sgs) fluctuations of the reactants and the temperature. A method of accounting for these is from the joint scalar probability density function $(p d f)$ of all the relevant scalar quantities which provides all the necessary information required to evaluate the filtered chemical source terms.

The modelled form of the equation governing the time evolution of the joint $p d f$ of the complete set of scalars provides a means of determining all of the time and spatially varying one-point statistics. The chemical source terms appear in closed form in this equation and no further modelling is required, beyond specification of a chemical reaction mechanism. Due to the high dimensionality of the $p d f$ evolution equation, a solution only becomes feasible if stochastic methods are applied. Conventionally Lagrangian stochastic particle methods have been adopted for the $p d f$ equation in conjunction with an Eulerian formulation for the velocity and pressure fields. Alternatively, Eulerian approaches have been formulated (see [1] and [2]). These methods introduce stochastic fields, which form a system of stochastic partial differential equations having the same one-point moments as the modelled $p d f$ evolution equation, Gardiner [3]. A main advantage of the latter method is that the solutions give rise to fields that are continuous and differentiable in space and which are thus free of spatially varying stochastic errors.

The application of LES to gas turbine combustors is a subject of much current interest. Previous studies have included, for example, LES of a swirl burner, [4] using a two parameter flamelet combustion model and LES of combustion instabilities in a lean-partially premixed combustor,[5] using a two-step global mechanism. More detailed chemical reaction mechanisms have also been incorporated, for example in the LES of a non-premixed, temporally evolving, syngas/air flame using an 11-species, 21-step mechanism in conjunction with an artificial neural networks approach, $[6]$. 


\section{Specific Objectives}

The LES-pdf formulation in conjunction with the Eulerian stochastic field solution method has been successfully applied in a range of burning configurations: ignition [7, 8] and auto-ignition [9], [10], nonpremixed [11] and premixed regimes [12]. The majority of cases were at atmospheric pressure and in relatively simple geometrical configurations. The work described in this paper aims to validate the LES$p d f$ method for an industrial gas turbine combustor (Siemens SGT-100), 0.3 MW thermal power operating at a pressure of 3 bar. Although the geometrical features of the combustion chamber were retained, the operating conditions (pressure, temperature and mixture fractions) studied and discussed in this paper differ from those of the burner in the SGT-100 gas turbine.

A SGT-100 combustor, shown in Fig. (1), was studied experimentally in the high pressure facility at the German Aerospace Center (DLR) Stuttgart, Germany [13], [14] and [15]. For ease of optical access the combustion chamber was modified so that its cross-section was square. Planar Induced Velocimetry (PIV), 1D Raman and OH PLIF data were collected from the burner.

\section{Large Eddy Simulation}

In LES the large scale energetic motions are computed directly with the effects of the unresolved sub-grid scale motions being modelled. Applying a density weighted filter to the conservation equations of mass and momentum yields:

Continuity:

$$
\frac{\partial \bar{\rho}}{\partial t}+\frac{\partial \bar{\rho} \tilde{u}_{i}}{\partial x_{i}}=0
$$

Momentum:

$$
\frac{\partial \bar{\rho} \tilde{u}_{i}}{\partial t}+\frac{\partial \bar{\rho} \tilde{u}_{i} \tilde{u}_{j}}{\partial x_{j}}=-\frac{\partial \bar{p}}{\partial x_{i}}+\frac{\partial}{\partial x_{j}} \bar{\sigma}_{i j}-\frac{\partial}{\partial x_{j}} \tau_{i j}
$$

where $\sigma_{i j}$ is the viscous stress tensor. The sub-grid scale stress tensor $\tau_{i j}=\bar{\rho}\left(\widetilde{u_{i} u_{j}}-\tilde{u}_{i} \tilde{u}_{j}\right)$ is determined via the dynamic version of the Smagorinsky model proposed by Piomelli and Liu [16]. The filtered equations for the mass fractions of chemical species contain sub-grid fluxes and filtered chemical source terms, which represent the filtered net rate of formation and consumption of the chemical species, as unknowns. A sub-grid $p d f$ is utilised to overcome the difficulties posed, in particular in the evaluation of the latter terms. 


\subsection{Sub-grid joint pdf}

An exact equation describing the evolution of the joint sub-grid (or more strictly the filtered fine grained) $p d f, \widetilde{P}_{\text {sgs }}$ can be derived by standard methods, eg. [17]. This equation contains unknown terms, representing $s g s$-transport of $p d f$ and sgs micro-mixing. In the present work these are represented, respectively, by a Smagorinsky type gradient model and by the Linear Mean Square Estimation (LMSE) closure, [18]. With these models incorporated the joint $-p d f$ equation for the $N$ scalar quantities needed to describe reaction can be written:

$$
\begin{aligned}
\bar{\rho} \frac{\partial \widetilde{P}_{s g s}(\underline{\psi})}{\partial t}+\bar{\rho} \tilde{u}_{j} \frac{\partial \widetilde{P}_{s g s}(\underline{\psi})}{\partial x_{j}}-\sum_{\alpha=1}^{N} \frac{\partial}{\partial \psi_{\alpha}}\left[\bar{\rho} \dot{\omega}_{\alpha}(\underline{\psi}) \widetilde{P}_{s g s}(\underline{\psi})\right]= \\
\frac{\partial}{\partial x_{i}}\left[\left(\left(\frac{\mu}{\sigma}+\frac{\mu_{s g s}}{\sigma_{s g s}}\right) \frac{\partial \tilde{P}_{s g s}(\underline{\psi})}{\partial x_{i}}\right)\right]-\frac{\bar{\rho}}{\tau_{s g s}} \sum_{\alpha=1}^{N} \frac{\partial}{\partial \psi_{\alpha}}\left[\left(\psi_{\alpha}-\phi_{\alpha}(\mathbf{x}, t)\right) \tilde{P}_{s g s}(\underline{\psi})\right]
\end{aligned}
$$

where $\sigma_{s g s}$ is assigned the value 0.7 and $\dot{\omega}_{\alpha}(\underline{\psi})$ is, in the case of chemical species the net formation rate through chemical reaction. The number of scalar quantities, $N$ is equal to the number of chemical species considered plus one (enthalpy). The micro-mixing time scale is obtained from $\tau_{s g s}{ }^{-1}=C_{d} \frac{\mu+\mu_{s g s}}{\bar{\rho} \Delta^{2}}$, where $C_{d}=2$.

\subsubsection{Eulerian stochastic field method}

The equation describing the evolution of the $p d f$, equation (3) is solved using the Eulerian stochastic field method. $\tilde{P}_{s g s}(\underline{\psi})$ is represented by an ensemble of $N_{s}$ stochastic fields with each field comprising the $N$ scalars, namely $\xi_{\alpha}^{n}(\mathbf{x}, t)$ for $1 \leq n \leq N_{s}, 1 \leq \alpha \leq N$. In the present work the Itô formulation of the stochastic integral is adopted and the stochastic fields thus evolve according to:

$$
\mathrm{d} \xi_{\alpha}^{n}=-\tilde{u}_{i} \frac{\partial \xi_{\alpha}^{n}}{\partial x_{i}} \mathrm{~d} t+\frac{\partial}{\partial x_{i}}\left[\Gamma^{\prime} \frac{\partial \xi_{\alpha}^{n}}{\partial x_{i}}\right] \mathrm{d} t+\dot{\omega}_{\alpha}^{n}\left(\underline{\xi}^{n}\right) \mathrm{d} t+\left(2 \Gamma^{\prime}\right)^{1 / 2} \frac{\partial \xi_{\alpha}^{n}}{\partial x_{i}} \mathrm{~d} W_{i}^{n}-\frac{1}{2 \tau_{s g s}}\left(\xi_{\alpha}^{n}-\tilde{\phi}_{\alpha}\right) \mathrm{d} t
$$

where $\Gamma^{\prime}$ represents the total diffusion coefficient and $d W_{i}^{n}$ represent increments of a (vector) Wiener process, different for each field but independent of the spatial location $\mathbf{x}$. This stochastic term has no influence on the first moments (or mean values) of $\xi_{\alpha}^{n}$. The stochastic fields given by (4) are not to be mistaken with any particular realization of the real field, but rather form an equivalent stochastic system (both sets have the same one-point $p d f,[3])$ smooth over the scale of the filter width. For more information about the Eulerian stochastic field method for combustion see $[8,9,10,11,12]$. The chemical reaction of methane-air mixtures is described by a GRI 3.0 reduced mechanism, [19] involving fifteen reaction steps and nineteen species. As 
a consequence with the inclusion of enthalpy twenty equations are solved for each field. Following [9] the number of stochastic fields was eight. The same mesh is used for both the LES and stochastic field equations (4).

\section{Computational Details}

The results to be presented below were obtained using the in-house block-structured, parallel, boundary conforming coordinate LES code, BOFFIN-LES [20]. The code has been applied to an extensive range of flows; further details of method can be found in, for example, [21].

The actual SGT-100 Dry Low Emission (DLE) combustor operating at 3 bar pressure conditions was selected as a test case. A structured mesh with 8 million cells and 240 blocks was generated using ICEMCFD. The solution domain comprises the radial burner, combustion chamber and a transition duct into an exhaust pipe. The solution domain includes the air inlet, fuel injection holes, a panel air inlet (experimental leakage) and the outlet. The fuel (German Natural Gas of $96.97 \% \mathrm{CH}_{4}$ ) with a temperature of $319.8 \mathrm{~K}$ was injected through multiple holes located in the swirler vanes. The main combustion air with a temperature of $685.3 \mathrm{~K}$ enters the combustor with a bulk velocity of $4.87 \mathrm{~m} / \mathrm{s}$. A minimum of 9 cells were used across the fuel injection holes whilst the majority contained 24 cells. The turbulence is generated by the swirler vanes, so no artificial turbulence was specified at the inlet boundary. The Reynolds number based on the inlet diameter of $0.19 \mathrm{~m}$ is 118,000 . The overall mixture fraction of the flame, including the panel air inlet is 0.0374 (0.0343 without panel air) compared with a stoichiometric value of 0.055 . All walls were treated as adiabatic and radiative heat transfer was neglected. At all solid boundaries wall-functions, based on the semi-logarithm law of the wall, [22] are applied as boundary conditions. In combustion chamber flows of the type considered the turbulence in the immediate vicinity of a solid surface and, indeed, the wall shear stress exert a negligible influence on the overall flow structure. The flow is dominated by turbulence generated far from walls. The major effect of the walls is simply to confine the flow.

After passing through the swirler vanes, the flow turns through a right angle into the prechamber, followed by a sudden expansion into the combustion chamber; the geometric swirl number, $S$, is 1.3. The flow in the combustion chamber exhibits three recirculation regions (i) an outer recirculation region formed in the wake of the burner exit and as a result of the combustor confinement; (ii) an inner recirculation region corresponding to the axisymmetric (bubble) vortex breakdown [23] and (iii) a weak central recirculation region dominated by the exit confinement. The inner reverse flow zone is attached to the back surface of the burner, thereby establishing a firm aerodynamic base for flame stabilisation. An $M$-shaped flame is stabilized 
in the shear layers between internal and external flow zones. The reaction layer thickness is around $0.05 \mathrm{~mm}$ and the thickness of the thermal layer is roughly ten times this.

\section{Results and Discussions}

The main results obtained from the SGT-100 burner are presented and discussed in this section. The flow field was allowed to 'settle' prior to the collection of statistical data. The total simulation time prior to collection of statistics but after burning was established was $50 \mathrm{~ms}$, which corresponds to about 10 burner flow-though times. Statistics were collected over approximately 10 flow through times. The time step was $5 \times 10^{-7} \mathrm{~s}$, the total number of time-steps was about 600,000 and the cost of the simulation was $19,750 \mathrm{CPU}$ hours The comparison of the LES results with experimental data was carried out at four axial locations in the combustor $(x / D=1.21,1.44,1.66,2.00$ where $D=0.086 \mathrm{~m}$ is the burner exit diameter), as depicted in Figure 2. Several points located in the flame region have been selected for study of the time evolution of temperature and species concentrations. An averaged profile of the mean $\mathrm{OH}$ molar concentration is also presented in Figure 2 to illustrate the flame position in respect to measurement profiles including 6 points selected for detailed analysis.

Figure 3 presents a comparison of PIV measured and simulated mean and RMS axial velocity at four different locations shown in Figure 2. The LES results agree well with the measured main flow field, as seen in Figure 3. Both (inner and outer) recirculation zones are well captured and shear layer regions were correctly reproduced. The flow field is fully turbulent as is indicated by high RMS fluctuations of axial velocity with values of approximately $40 \%$ of the mean. Over all the level of agreement between the measured and simulated RMS profiles is reasonable, although the maximum simulated values are somewhat too low. The combustor walls determine both inner and outer recirculation zones. On the centre axis a weak region of forward axial flow acceleration corresponding to the weak central recirculation region is observed. This is in agreement with early experimental observations of confined swirling flows [24].

The time evolution of temperature and species has been collected and compared with experimental data $[13,14]$ at the locations identified in Figure 2. The comparison of the LES (left) and 1-D Raman (mid) results of scatter plots of temperature and mixture fraction is presented in Figure 4. The plots have been overlayed by lines indicating an adiabatic flame temperature and gas mixing lines computed by a flamelet calculation. Scaled histograms of mixture fractions are also included with each scatter plot. In the right column of Figure 4 the comparison of the temperature $p d f$ for the LES and 1D Raman is presented for each point. Good overall agreement for points inside the flame brush $(P 116)$ and in the inner shear layer 
(P113, P315) is noted. A slight over-estimation of temperature was observed in the flame tip (P519) and outer shear layer (P119). The maximum temperatures arising in the simulations are somewhat higher than the measured values, this being most evident at P119 where simulated maximum temperatures of around $2250 \mathrm{~K}$ are evident. This may well be due to the neglect of radiative hat transfer in the simulations. In case of the centre axis point $(P 100)$, where only fully reacting products exists, the peak temperature is captured well, but not the distribution around the peak. It is possible that this arises from the chemical mechanism used as the RMS levels of velocity are well captured as shown in Figure 3. Overall, small differences in scatter may also be attributed to experimental error or to differences in the data collection time, i.e. several minutes in the experiment and compared with several milliseconds in the computation.

Some evidence of local extinction is also present. Conditioning of the temperature with mixture fraction indicates the cause of the local extinction. When the conditioned temperature is constant the extinction is mainly caused by the mixing of large scale turbulent motions. However, the local reaction effects probably associated with flame stretch result in fluctuations in the conditioned temperature. To identify the extinction regions observed in Figure 4 the temperature was conditioned on a mixture fraction of $(f=0.03)$. Data from the 3 points at the first axial location and differing radial positions in the flame is presented in Figure 5 against time: time traces of temperature and mixture fraction were taken at three specific points. From these, values of temperature and time were extracted when the mixture fraction values lay within the interval $0.03 \pm 0.002$ Significant changes of temperature for the same mixture fraction indicate that the local extinction is due to chemistry/turbulence interactions. The frequency of such events corresponds (approximately) to large scale flow motions, which may be associated with vortex shedding. The frequency of local extinction is greater in the inner shear layer (P113) than on the outer shear layer (P119) and this is attributed to stronger shear-induced turbulence in the inner shear layer than in the outer. Similar extinction events have been captured with LES for Sandia flame $F,[11]$.

The flame index [25] has been computed as the product of the Methane and Oxygen mass fraction gradients and is presented in Figure 6 together with a contour of mixture fraction of 0.0343. The flame index is used to distinguish between the premixed and diffusion flame regimes with a positive and negative values corresponding to the premixed and diffusion flame regimes respectively. Figure 6 shows that most of the combustion occurs in a premixed regime, however there are regions inside the flame where diffusion conditions exist, for example. This clearly shows that an industrially premixed burner such as SGT-100 has reaction regions of a diffusion nature. This indicates that the major working regime of such a burner is partially premixed. The zero (green) values of the flame index corresponds to regions occupied by either air 
or fully burnt mixture.

An instantaneous time snapshot of the flame region in the front of the combustor is presented in Figure 7 for mixture fraction, temperature, $\mathrm{CO}, \mathrm{NO}$ and $\mathrm{OH}$ mass fractions and shows the complex flame structure captured by the LES-pdf model. Regions of local extinction and of local high temperatures are observed for very similar values of mixture fraction. Vortex engulfment of the flame is also well captured. The locations of regions of major heat release (presented as $\mathrm{OH}$ ) has been identified as well as regions of high $\mathrm{CO}$ concentration. The NO concentrations are relatively small, but are concentrated in small pockets in the flame region or in the immediate vicinity of the flame front. In the flame region, NO is formed in high heat release parts of the flame and is then transported downstream. The distribution of NO mass fraction with temperature is presented in figure 8 for the 6 points of interest. The data included in the scatter plot was obtained by sampling from each stochastic field at each time step at every $100^{\text {th }}$ node over the data collection time In the middle of figure 8 the $p d f$ of temperature is included whilst on the left is the $p d f$ of NO mass fraction is presented. It should be noted that the same bin width of $40 \mathrm{~K}$ has been used for temperature, whilst a bin width of $2 \times 10^{-7}$ was used for NO. The NO mass fraction data presented in figure 8 is multiplied to $10^{6}$ for clarity before plotting.

The NO formation rate is shown in figure 9 where the mean time averaged formation rate is shown in the form of a contour plot in a plane through the centre of the combustor; the upper plot shows the total NO formation rate whereas the lower plot shows the rate of formation of NO via the extended Zeldovich mechanism, i.e. thermal NO. As is evident NO is formed almost exclusively in a very small region of the combustion chamber in the high temperature flame brush zone, i.e. in the vicinity of $P 113, P 116, P 119$, P315 and P519. The presence of unburnt fuel-air mixture in this region indicate the presence of a flame front. The figure also shows that NO is formed as a combination of the thermal and prompt, [26] NO mechanisms. The maximum total rate of NO formation is $0.025 \frac{\mathrm{Kmol}}{\mathrm{kg} \mathrm{s}}$ compared $0.0066 \frac{\mathrm{Kmol}}{\mathrm{kg} \mathrm{s}}$ for the thermal NO; the prompt mechanism constitutes roughly $70 \%$ of the total rate.

The measured concentration of $\mathrm{NO}$ at the outlet plane was $12.1 \mathrm{ppmv}$ corrected to $15 \% \mathrm{O}_{2}$ and compared with a simulated level of $15.06 \mathrm{ppmv}$. Measurements were taken downstream of the exhaust at $30 \mathrm{~mm}$ and include a total of $7.5 \%$ air leakage. As air leakages were not included in the LES and the result was corrected to account for them. Overall, a good agreement was found between LES and measured concentrations. 


\section{Conclusions}

In summary Large Eddy Simulations with a sub-grid $p d f$ model with reduced chemistry has been successfully applied to an industrial gas turbine burner at pressure under laboratory conditions. The calculations were carried out using a detailed block-structured mesh capturing all geometrical features of the SGT-100 burner with the BOFFIN code. A sub-grid dynamic model and 19 species reaction from a 15-step mechanism were used.

Results obtained from the calculations lead to following conclusions:

- Good reproduction of the flow field was achieved for a highly swirling flow in a complex geometry with a dynamic sub-grid model.

- Accurate predictions of temperature and species in the inner shear layer were obtained.

- Local extinction of the flame was shown to be due to local chemistry/turbulence effects rather than large scale mixing.

- The frequency of the local extinction was greater in the inner shear layer than in the outer shear layer and was attributed to vortex shedding.

- The burner was found to have regions of diffusion combustion regimes, but mainly operates in a premixed regime.

- The measured emission levels of NO were reproduced to a good accuracy.

\section{Acknowledgement}

This work was supported by SIEMENS Industrial Turbomachinery Ltd. The support of Ulrich Stopper from DLR Stuttgart and Dr. Alessio Bonaldo from SIEMENS for help in processing the experimental data is greatly appreciated.

\section{References}

[1] L. Valino, Flow, Turbulence and Combustion 60 (2) (1998) 157-172.

[2] V. Sabel'nikov, O. Soulard, Physial Review E 72 (1) (2005) 016301.

[3] C. Gardiner, Handbook of Stochastic Methods, Springer Verlag, 1985.

[4] V. Moureau, P. Domingo, L. Vervisch, Combustion and Flame 158 (7) (2011) 1340-1357.

[5] B. Franzelli, E. Riber, L. Y. Gicquel, T. Poinsot, Combustion and Flame 159 (2) (2012) 621-637. 
[6] B. A. Sen, E. R. Hawkes, S. Menon, Combustion and Flame 157 (3) (2010) 566-578.

[7] W. P. Jones, A. Tyliszczak, Flow Turbulence Combust. 85 (2010) 711-734.

[8] W. P. Jones, V. N. Prasad, Proc Combust Inst 33 (2011) 1355-1363.

[9] W. P. Jones, S. Navarro-Martinez, Combust Flame 150 (3) (2007) 170-187.

[10] W. P. Jones, S. Navarro-Martinez, Computers 65 Fluids 37 (2007) 802-808.

[11] W. P. Jones, V. N. Prasad, Combust Flame 157 (9) (2010) 1621-1636.

[12] V. Prasad, Large Eddy Simulation of partially premixed turbulent combustion, Ph.D. thesis, Imperial College, University of London (2011).

[13] U. Stopper, M. Aigner, W. Meier, R. Sadanandan, M. Stör, I. Kim, J Engineering for Gas Turbines and Power 131 (2) (2009) 021504-1,021504-8.

[14] U. Stopper, M. Aigner, H. Ax, W. Meier, R. Sadanandan, M. Stör, A. Bonaldo, Experimental Thermal and Fluid Science 34 (3) (2010) 396-403.

[15] U. Stopper, Experimental results from TURCHEMI test campaign on SGT-100: DLR-Siemens internal database, Tech. rep., DLR, Stuttgart (2011).

[16] U. Piomelli, J. Liu, Phys Fluids 7 (4) (1995) 839-848.

[17] F. Gao, E. O’Brian, Phys Fluids A 5 (1993) 1282-1284.

[18] C. Dopazo, Phys Fluids 22 (1) (1979) 20-30.

[19] C. J. Sung, C. K. Law, J. Y. Chen, Combustion and Flame 125 (2001) 906-919.

[20] W. P. Jones, F. di Mare, A. J. Marquis, LES-BOFFIN: Users Guide, Technical Memorandum, Imperial College, London (2002).

[21] W. P. Jones, A. J. Marquis, V. N. Prasad, Combustion and FlameIn press.

[22] U. Piomelli, E. Balaras, Annual Review of Fluid Mechanics 34 (2002) 349-374.

[23] T. Sarpkaya, J Fluid Mech 45 (3) (1971) 545-559.

[24] N. Syred, J. M. Beér, Combust Flame 23 (1974) 143-201.

[25] Y. Mizobuchi, S. Tachibana, J. Shinio, S. Ogawa, T. Takeno, Proc Combust Inst 29 (1) (2002) $2009-2015$.

[26] C. Fenimore, Symposium (International) on Combustion 13 (1971) 373-380. 


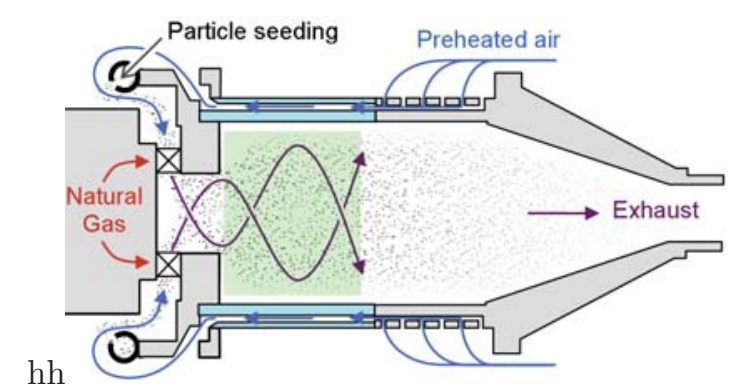

Figure 1: Experimental setup, [13], region of interest highlighted in light green.

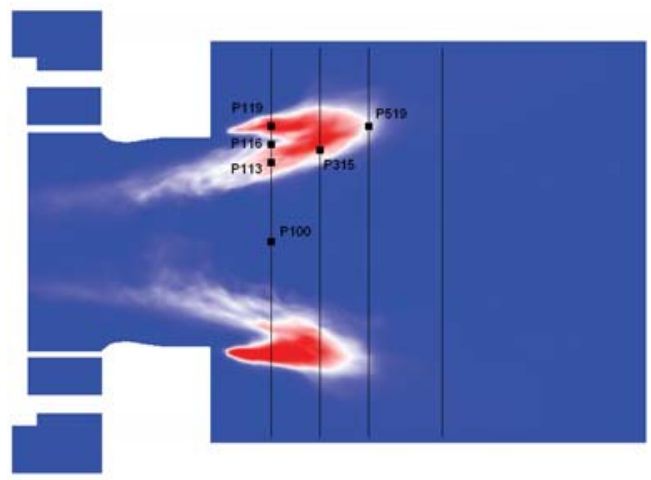

Figure 2: Profile of mean $\mathrm{OH}$ molar mass fraction and location of experimental points
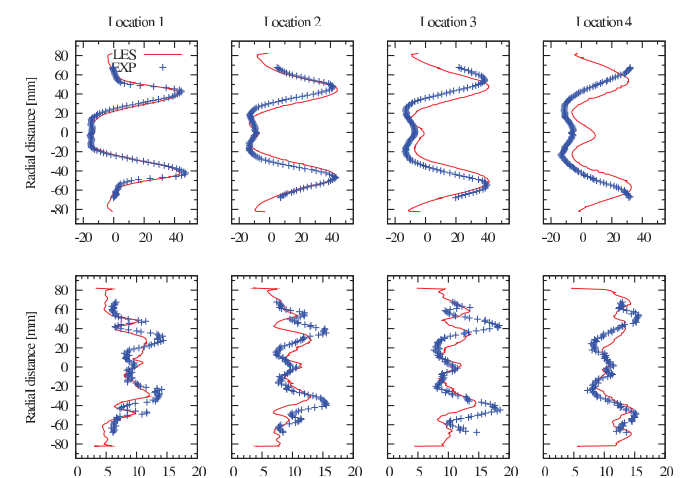

Figure 3: Comparison of mean (top) and RMS (bottom) Axial Velocity (m/s) profiles. 

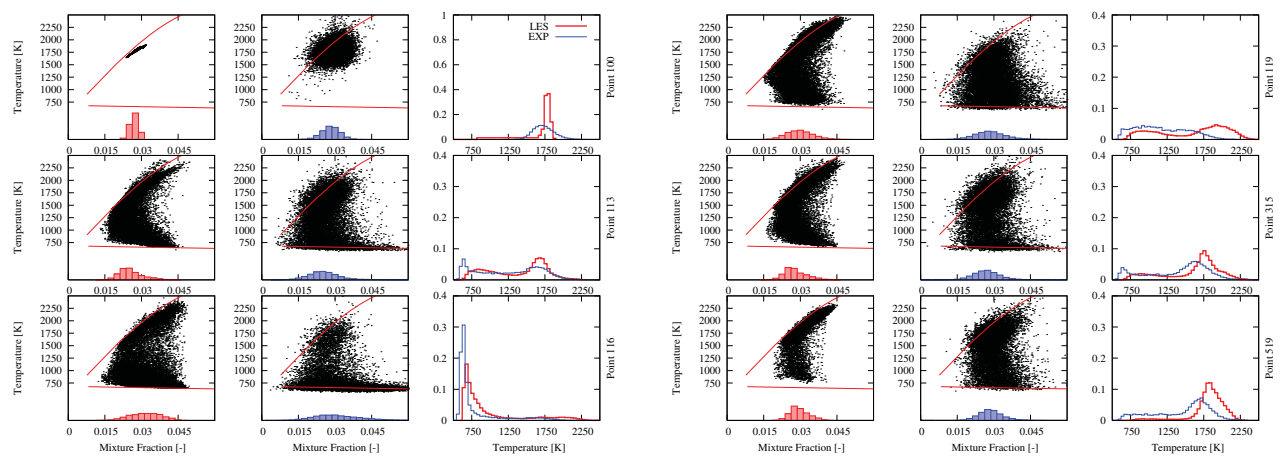

Figure 4: Comparison of Scatter Plots between LES (left) and 1-D Raman (mid), mixing lines; $p d f$ of temperature(right).

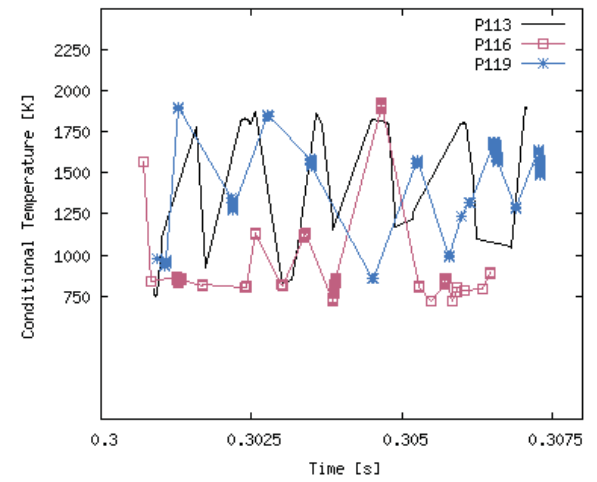

Figure 5: Time evolution of Conditioned Temperature to mixture fraction of $f=0.03$ at different locations in the flame.

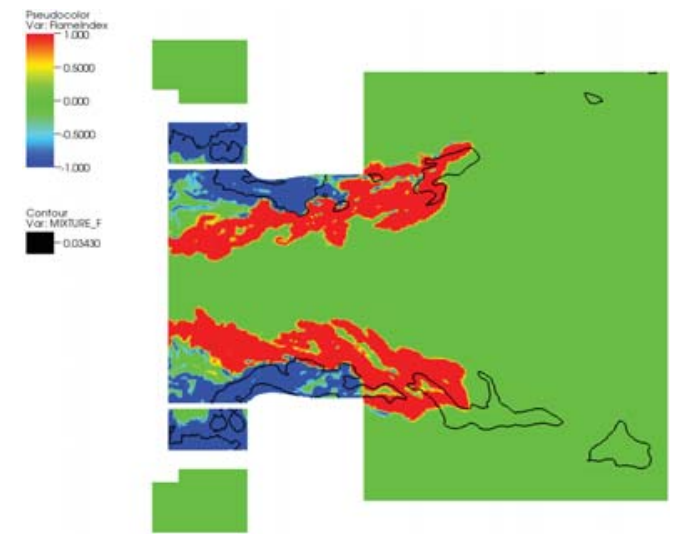

Figure 6: Flame index [25] with a contour of the overall burner mixture fraction $f=0.0343$. 


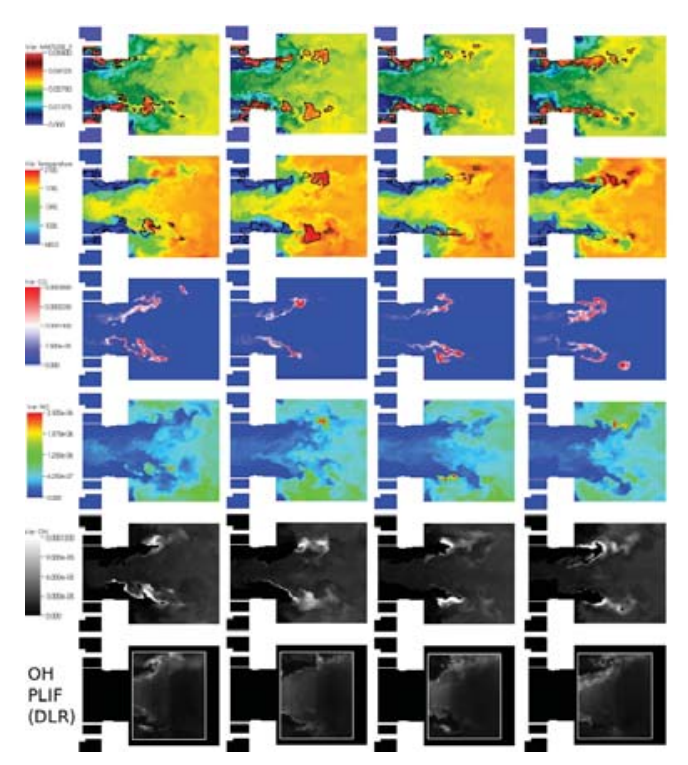

Figure 7: Instantaneous snapshots of mixture fraction (top), temperature (K), $\mathrm{CO}, \mathrm{NO}$ and $\mathrm{OH}$ mass fraction concentrations and the experimental OH PLIF, [15] (bottom)

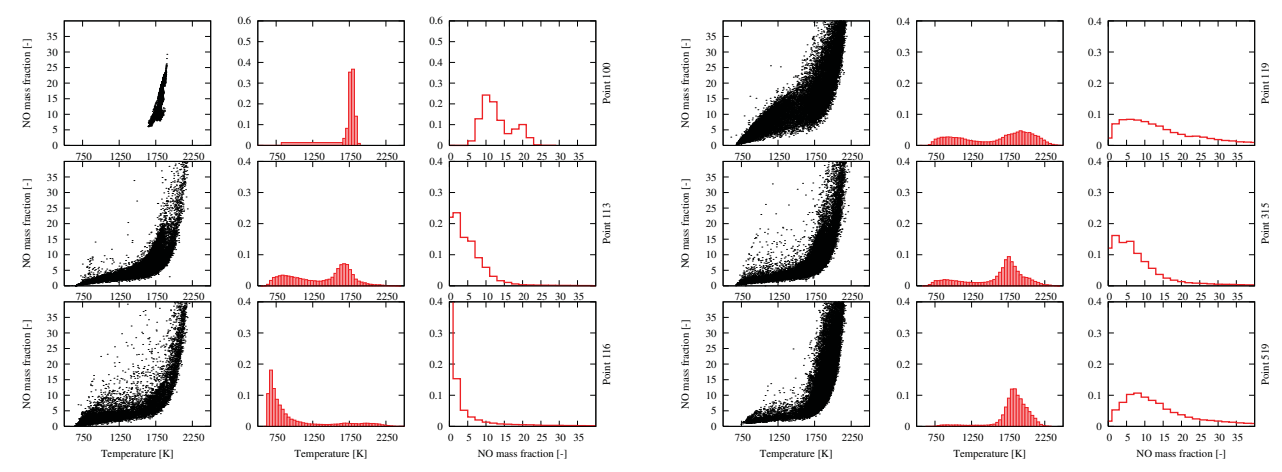

Figure 8: Scatter plot of NO Mass Fraction vs temperature (left) and $p d f$ of temperature (mid) and $p d f$ of NO mass fraction (right). Note that $\mathrm{NO}$ values have been scaled by $10^{6}$

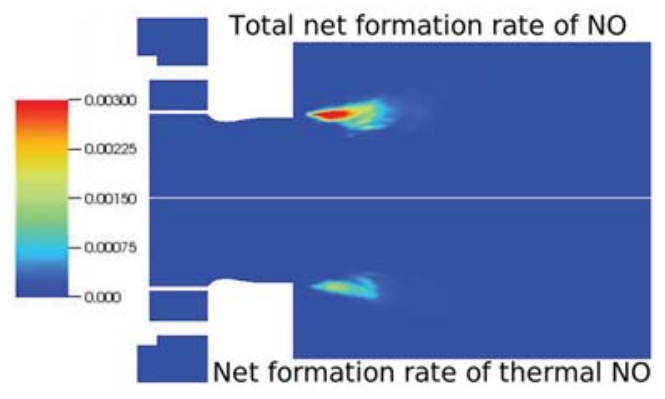

Figure 9: Time averaged NO formation rates, $\frac{\mathrm{Kmol}}{\mathrm{kgs}}$ 


\section{List of Figures}

1 Experimental setup, [13], region of interest highlighted in light green. . . . . . . . . . . . . . 11

2 Profile of mean $\mathrm{OH}$ molar mass fraction and location of experimental points . . . . . . . . . 11

3 Comparison of mean (top) and RMS (bottom) Axial Velocity (m/s) profiles. . . . . . . . . . 11

4 Comparison of Scatter Plots between LES (left) and 1-D Raman (mid), | adiabatic flamelet temperature and mixing lines; $p d f$ of temperature(right). . . . . . . . . . . . . . . . . 12

5 Time evolution of Conditioned Temperature to mixture fraction of $f=0.03$ at different locations in the flame. . . . . . . . . . . . . . . . . . . . . . 12

6 Flame index [25] with a contour of the overall burner mixture fraction $f=0.0343 . \quad$. . . . 12

7 Instantaneous snapshots . . . . . . . . . . . . . . . . . . . . . . 13

8 Scatter plot of NO Mass Fraction vs temperature . . . . . . . . . . . . . . . 13

9 Time averaged $\mathrm{NO}$ formation rates, $\frac{\mathrm{Kmol}}{\mathrm{kg} \mathrm{s}} \ldots \ldots \ldots \ldots \ldots \ldots$ 